Leonard Pon | Sveučilište Josipa Jurja Strossmayera u Osijeku, Filozofski fakultet, Ipon@ffos.hr Ana Keglević | Osnovna škola kralja Tomislava, Našice, keglevic.ana@gmail.com

\title{
Zur Grammatikkompetenz kroatischer DaF-Lernender nach Abschluss der Mittelschule
}

\section{Einführung und Fragestellung}

Im vorliegenden Beitrag widmen wir uns der Analyse der Grammatikkompetenz kroatischer DaF-Lernender. ${ }^{1}$ Kroatische Lernende setzen sich in der Mittelschule im Rahmen einer expliziten Wissensvermittlung mit vielen syntaktischen Phänomenen auseinander. Darüber hinaus werden während dieser Zeit auch andere Arten von Spracharbeit gepflegt, bei denen Lernende ihr grammatisches Wissen implizit erweitern können. Doch mit welchem Ergebnis? Im vorliegenden Beitrag möchten wir auf empirischem Wege herausfinden, über welche grammatischen Kenntnisse kroatische DaF-Lernende nach Abschluss der Mittelschule verfügen. Wir wollen uns dabei auf ihre Syntaxkenntnisse konzentrieren. Zu diesem Zweck werden freie schriftliche Produktionen einer Lernendengruppe analysiert und die so gewonnenen Ergebnisse u.a. unter Heranziehung der Unterrichtspläne interpretiert.

1 Die vorliegende Arbeit ist im Rahmen des Projekts »Aspekte der Grammatikkompetenz kroatischer DaF-Lernender « entstanden, das seit März 2015 von der Josip-Juraj-StrossmayerUniversität in Osijek finanziell unterstützt wird.
Im vorliegenden Beitrag wird nach der Grammatikkompetenz kroatischer DaF-Lernender nach bestandener Abiturprüfung im Fach Deutsch (höhere Ebene) gefragt, da der Grammatikvermittlung verhältnismäßig viel Unterrichtszeit gewidmet und von diesem Unterricht auch viel erwartet wird. Zur Beantwortung der Forschungsfragen werden schriftliche Produktionen der Lernenden unter Heranziehung der Profilanalyse untersucht. Eine differenzierte Einsicht in die Grammatikkompetenz der Probanden erfolgt einerseits aufgrund quantitativer Ergebnisse (u.a. Korrelationsanalyse), andererseits aufgrund qualitativer Beobachtungen sowie einer Analyse ausgewählter Belege. 
An vielen kroatischen Schulen ist Deutsch die zweitwichtigste Fremdsprache. Im Grunde genommen besteht das Ziel des Deutschunterrichts in Kroatien darin, die Lernenden zu befähigen, Deutsch in verschiedenen kommunikativen Situationen angemessen zu verwenden. Um dieses Ziel zu erreichen, werden im Deutschunterricht u.a. grammatische Regeln erklärt und geübt. Da sehr viele Stunden gerade auf Grammatikstunden entfallen, ist es unerlässlich, die Frage nach dem Sinn und Erfolg des Grammatikunterrichts zu stellen. Anders ausgedrückt: Leistet unser Grammatikunterricht tatsächlich den erwarteten Beitrag?

Bezüglich der Stufe und der Kenntnisse, die die Abiturienten in Kroatien aufweisen sollen, existieren einerseits Lehrpläne für die Mittelstufe, in denen angegeben wird, was vor dem Abitur beherrscht werden soll. Andererseits liegt ein Katalog vor, der detailliert beschreibt, über welches Wissen Abiturienten verfügen sollten. ${ }^{2}$ Nach diesem Katalog entspricht die Abiturprüfung im Fach Deutsch (höhere Ebene) der Niveaustufe B2 gemäß dem Gemeinsamen europäischen Referenzrahmen für Sprachen (weiter: GERS).

Im vorliegenden Beitrag wird versucht, die Grammatikkompetenz einer Gruppe kroatischer DaF-Lernender zu untersuchen. Zu diesem Zweck haben die Probanden nach bestimmten Vorgaben schriftliche Produktionen verfasst, die im Nachhinein analysiert wurden. Die Probanden befanden sich zum Zeitpunkt der Datenerhebung gerade an einer Schnittstelle: Sie haben die Mittelschule abgeschlossen und standen am Anfang ihres Studiums. Es ist durchaus nützlich, die Deutschkenntnisse der Lernenden in dieser Phase näher zu beleuchten, weil dabei deutlich zutage tritt, inwieweit die Ziele des Deutschunterrichts tatsächlich erreicht werden.

\section{Status und Entwicklung der Grammatikkompetenz}

Forscher aus dem Bereich der Angewandten Linguistik haben in den vergangenen Jahren mehrere Modelle zur Beschreibung der kommunikativen Kompetenz ${ }^{3}$ entwickelt. Alle diese Modelle enthalten u.a. eine Komponente oder Subkomponente, die mit dem Terminus Grammatikkompetenz oder

2 Vgl. Ispitni katalog, S. 5 f.

3 Vgl. Canale/Swain: Theoretical Bases of Communicative Approaches to Second Language Teaching and Testing; Canale/Swain: A Theoretical Framework for Communicative Competence; Cohen: Assessing Language Ability in the Classroom; Savignon: Communicative Competence; Bachmann/ Palmer: The Construct Validation of Some Components of Communicative Proficiency; Bachmann/ Palmer: Language Testing in Practice; Bachmann/Palmer: Language Assessment in Practice; 
Sprachkompetenz bezeichnet werden kann. Die Analyse dieser Modelle zeigt, dass die Kenntnis der grammatischen Regeln einer Fremdsprache nicht mit der kommunikativen Kompetenz gleichgesetzt werden darf, dass aber diese Kenntnisse als eine unabdingbare Voraussetzung für erfolgreiches Kommunizieren in der betreffenden Fremdsprache angesehen werden. Im Zusammenhang mit den genannten Modellen möchten wir zwei wichtige Tatsachen nennen. Erstens geht das Konzept der Grammatik- bzw. Sprachkompetenz über morphologische und syntaktische Kenntnisse hinaus und umfasst auch phonologische, lexikalische, bisweilen auch orthographische Kenntnisse. Zweitens reden einige Forscher dabei einfach von Kenntnissen, ${ }^{4}$ während andere den Aspekt der Anwendung hervorheben. Dementsprechend betont Savignon, dass sich die Grammatikkompetenz der Sprachbenutzer in ihrer Fähigkeit der Anwendung grammatischer Regeln manifestiert. ${ }^{5}$

Nach dem GERS besteht die kommunikative Kompetenz aus linguistischen, soziolinguistischen und pragmatischen Kompetenzen. ${ }^{6}$ Die linguistischen Kompetenzen umfassen lexikalische, grammatische, semantische und phonologische Kompetenz. ${ }^{7}$ Dabei definiert sich die grammatische Kompetenz »als Kenntnis der grammatischen Mittel einer Sprache und die Fähigkeit, diese zu verwenden «. ${ }^{8}$

Einige Studien haben gezeigt, dass die explizite Vermittlung grammatischer Regeln samt anschließenden Übungen nicht immer zu Äußerungen führt, die grammatisch richtig sind. ${ }^{9}$ Dies hängt mit dem Verhältnis des expliziten und impliziten Wissens zusammen. Dabei geht es um die häufig angesprochene Frage, ob das explizite Wissen in das implizite transferiert werden kann. ${ }^{10}$ Um diese Frage angemessen zu beantworten, sind empirische Beweise erforderlich. Diehl und ihre Mitarbeiter konnten in einem breit angelegten Projekt feststellen, dass zwischen den im Grammatikunterricht angestrebten Zielen und der realen Grammatikkompetenz der

Celce-Murcia/Dörnyei/Thurrell: Communicative Competence; Bagarić Medve: Komunikacijska kompetencija.

4 Vgl. Canale/Swain: Theoretical Bases of Communicative Approaches to Second Language Teaching and Testing; Canale/Swain: A Theoretical Framework for Communicative Competence.

5 Vgl. Savignon: Communicative Competence.

6 Vgl. GERS, S. 109.

7 Ebd., S. 110.

8 Ebd., S. 113.

9 Zur Frage nach den Möglichkeiten und Grenzen der expliziten Grammatikvermittlung sowie zu den Faktoren, die die Grammatikkenntnisse der Fremdsprachenlernenden beeinflussen, vgl. Pon: Grammatisches Wissen, S. 38f.

10 Vgl. Ellis: Current Issues in the Teaching of Grammar; ders.: Principles of Instructed Second Language Acquisition. 
DaF-Lernenden eine große Kluft besteht. ${ }^{11}$ Viele Strukturen, die im Rahmen des expliziten Grammatikunterrichts behandelt wurden, konnten bei den Lernenden nicht als erworben nachgewiesen werden. Es konnte ferner festgestellt werden, dass bestimmte grammatische Phänomene immer in einer Abfolge (d.h. Sequenz) erworben werden. Der Erwerb dieser Phänomene kann durch explizite Grammatikunterweisung nicht beschleunigt werden. Die einzelnen Sequenzen kommen generell bei allen Lernenden vor, egal ob wir es mit dem ungesteuerten oder gesteuerten Erwerb zu tun haben. Für die vorliegende Arbeit ist die Erkenntnis wichtig, dass der Syntaxerwerb allem Anschein nach gewisse Sequenzen aufweist, deren Abfolge nicht verändert werden kann.

Einige Autoren verweisen auf die Notwendigkeit, verschiedene Sprachenpaare zu analysieren, weil die L $1^{12}$ als Faktor eine große Rolle spielt. Zudem zeigt Sanchez anhand des Erwerbs der englischen Wortstellung, dass nicht nur die L1, sondern alle anderen Sprachen, die ein Lernender beherrscht, beim Erlernen einer weiteren Fremdsprache eine Rolle spielen: »This evidence suggests that the arrangement of the object and the verb is heavily constrained by the $\mathrm{OV}$ or $\mathrm{VO}$ sequence of languages in the linguistic repertoire of the learner $[. ..] .{ }^{13}{ }^{13}$ Konkret konnte Sanchez nachweisen, dass neben Spanisch und Katalanisch, die bei ihren Lernenden als L1 auftraten, auch die L3 - hier Deutsch - für den Transfer beim Erwerb der englischen Wortstellung verantwortlich ist.

Da sich Grammatikkompetenz, insbesondere Syntaxkompetenz, sequentiell entwickelt, wäre es aus theoretischer und praktischer Sicht höchst notwendig, empirisch zu erforschen, welchen Entwicklungsstand DaFLernende an wichtigen Schnittstellen aufweisen (z.B. beim Schul- oder Hochschulabschluss), wenn ihre Muttersprache Kroatisch und ihre erste Fremdsprache in der Regel Englisch ist. Einigen Aspekten, die mit Syntaxkompetenz zusammenhängen, widmet sich die vorliegende Untersuchung.

\section{Untersuchung}

\subsection{Ziel, Probanden und Korpus}

Das Ziel der vorliegenden Arbeit besteht darin, das Niveau der Grammatikkompetenz zu beschreiben, wie es für kroatische DaF-Lernende nach

11 Vgl. Diehl: Schulischer Grammatikerwerb unter der Lupe; Diehl et al.: Grammatikunterricht.

12 Die Abkürzung L1 steht für Muttersprache, L3 für zweite Fremdsprache.

13 Sanchez: Against >Canonical Word Order, S. 229. 
Abschluss der Mittelschule kennzeichnend ist. Die Forschungsfragen, die diese Arbeit beantworten sollte, lauten:

1. Welches Niveau der Grammatikkompetenz haben die Probanden erreicht?

2. In welchem Zusammenhang stehen die Ergebnisse zweier verschiedener Verfahren zur Ermittlung der Grammatikkompetenz zueinander (ECL-Test vs. Profilanalyse)?

3. In welchem Zusammenhang steht die erreichte Stufe der Grammatikkompetenz unserer Probanden zu den offiziell festgelegten und erwünschten Zielen des Fremdsprachenunterrichts für die betreffende Probandengruppe?

Unsere Probanden sind 57 Lernende, die im Oktober 2014 ihr Germanistikstudium in Osijek angefangen haben. Ihre Gemeinsamkeit besteht darin, dass sie die Abiturprüfung im Fach Deutsch (auf der sog. höheren Ebene) bestanden und dabei mindestens 60\% der maximalen Punktezahl erreicht haben. Dies entspricht offiziell der Niveaustufe B2 des Gemeinsamen europäischen Referenzrahmens für Sprachen. ${ }^{14}$ Da die Probanden aus verschiedenen Regionen Kroatiens stammen und nur eine Gemeinsamkeit haben - die Immatrikulation in dasselbe Studium und die erfolgreich bestandene höhere Ebene der Abiturprüfung im Fach Deutsch -, stellen sie eine durch Zufallsauswahl gebildete Probandengruppe dar.

Als Korpus dienen schriftliche Produktionen dieser Probanden, die im Rahmen eines ECL-Tests entstanden sind (s. 3.2).

\subsection{Instrumente}

Als Instrumente im empirischen Teil dienten ein Test zur Schreibfertigkeit und ein Raster, mit dem u.a. die formelle Korrektheit schriftlicher Produktionen bewertet wird.

Im vorliegenden Beitrag werden schriftliche Produktionen untersucht, die im Rahmen des im Oktober 2014 durchgeführten ECL-Tests entstanden sind. Alle Probanden haben vor dem Beginn des universitären Unterrichts an einer vom ECL-Zentrum in Pécs durchgeführten Prüfung teilgenommen, in deren Rahmen u.a. eine schriftliche Produktion verfasst wurde. Diese Produktionen bilden das Korpus der vorliegenden Analyse. Nach konkreter

14 Vgl. Ispitni katalog, S. 5-7. Hierbei geht es um einen Katalog für die Abiturprüfung in deutscher Sprache, in dem u.a. grammatische Strukturen verzeichnet sind, die kroatische DaF-Lernende beim Abschluss der Mittelschule beherrschen sollen. 
Anweisung sollten die Probanden eine ungefähr 125 Wörter umfassende Nachricht für ihren Freund verfassen und dabei auf fünf verschiedene Punkte eingehen. Die zu behandelnden Punkte sind in der Anweisung genau beschrieben.

Zur Bewertung schriftlicher Produktionen wurde ein Raster angewendet, das sich auf folgende Aspekte bezieht: formelle Korrektheit, schriftliche Korrektheit, Wortschatz, Stil und kommunikative Effektivität. Für jeden Aspekt können 1 bis 5 Punkte vergeben werden, den Bewertern stehen dabei Deskriptoren zur Verfügung. Wir interessieren uns hier für den ersten Aspekt - formelle Korrektheit, denn er umfasst Morphologie und Syntax. Die Bewertung wurde vom ECL-Personal durchgeführt und uns wurden die Ergebnisse zur Verfügung gestellt. Wichtig zu betonen wäre, dass es sich hierbei um geschulte Bewerter handelt, deren hauptsächliche Aufgabe gerade darin besteht, Testaufgaben zu entwickeln und Tests auf verschiedenen Stufen zu bewerten.

\subsection{Methode}

Die zur Ermittlung der Grammatikkompetenz herangezogene Methode ist die Profilanalyse, wie sie von W. Grießhaber durchgeführt wird. ${ }^{15}$

Die Profilanalyse wird als ein Verfahren beschrieben, das eine schnelle Sprachstandsermittlung von Fremdsprachenlernenden ermöglicht. Sie beschränkt sich eigentlich auf den syntaktischen Bereich. Es konnte nämlich nachgewiesen werden, dass der Syntaxerwerb bzw. der Erwerb der Stellungsregularitäten sequentiell verläuft. Jeder Lernende erwirbt bspw. die Stellung des Verbpräfixes am Ende eines Aussagesatzes, erst nachdem er gelernt hat, dass es in jedem Satz ein finites Verb gibt. Übrigens sei angemerkt, dass die Stufen, von denen im vorliegenden Beitrag die Rede ist, sprachspezifisch sind: sie gelten für Deutsch. ${ }^{16}$ Wir übernehmen hier das Instrumentarium, wie es Grießhaber in einer Reihe seiner Veröffentlichungen verwendet.

Das Verfahren der Profilanalyse besteht aus drei Schritten. Zunächst wird der zu analysierende Text in minimale satzwertige Einheiten zerlegt, die wir mit dem Terminus Teilsatz bezeichnen könnten. Syntaktisch gesehen, handelt es sich dabei um einen einfachen Satz, einen Hauptsatz oder einen Nebensatz. Im Aufsatz eines Probanden steht Folgendes:

15 Grießhaber: Zum Verfahren der Sprachprofilanalyse; ders.: Sprachstandsdiagnose im kindlichen Zweitspracherwerb; ders.: Beurteilung von Texten mehrsprachiger Schülerinnen und Schüler.

16 Vgl. Grießhaber: Zum Verfahren der Sprachprofilanalyse. 
Inzwischen werde ich einkaufen gehen und dir Schinken, Salaten, Milch und Eier im Kühlschrank lassen, sodass, wenn du kommst, etwas zum Essen hast. In der Kuche befinden sich alle Haushaltsgeräte, die du fürs Kochen brauchst. Bügeleisen werde ich in Wohnzimmer lassen und eine Waschmaschine wirst du in dem Bad finden.

Zerlegen wir diesen Text, erhalten wir acht minimale satzwertige Einheiten:

Inzwischen werde ich einkaufen gehen / und dir Schinken, Salaten, Milch und Eier im Kühlschrank lassen, / sodass, (wenn du kommst), ${ }^{17}$ etwas zum Essen hast. / wenn du kommst / In der Kuche befinden sich alle Haushaltsgeräte, / die du fürs Kochen brauchst. / Bügeleisen werde ich in Wohnzimmer lassen / und eine Waschmaschine wirst du in dem Bad finden.

Im Anschluss an diesen ersten Schritt soll jede Einheit einer der Stufen zugeordnet werden. An dieser Stelle werden die einzelnen Stufen vereinfacht dargestellt, Grießhaber liefert viele Beispiele und umfassendere Hinweise zur Durchführung der Analyse. ${ }^{18}$

Unter Stufe 0 werden bruchstückhafte Äußerungen verstanden, die häufig keine Verben enthalten (z.B. Hallo, mein Freund). Stufe 1 bedeutet, dass eine Äußerung ein finites Verb an zweiter Stelle enthält (z.B. die Waschmaschine befindet sich im Badezimmer). Häufig enthalten solche Äußerungen nur ein Subjekt und ein Verb. Stufe 2 bedeutet, dass im Satz ein mehrteiliges Prädikat vorkommt und dass dabei alle Verbteile an korrekten Stellen im Satz erscheinen: das finite Verb steht an zweiter, das infinite an letzter Stelle im Satz. Mit der Stufe 3 werden Äußerungen bezeichnet, die an erster Stelle ein Element außer dem Subjekt enthalten und an zweiter das finite Verb. In solchen Äußerungen kann also die sog. Inversion beobachtet werden (z.B. und eine Waschmaschine wirst du in dem Badfinden). Die Stufe 4 bezieht sich auf die Nebensatzstellung: ein Subjunktor steht am Anfang, das finite Verb an letzter Stelle (z.B. die du fürs Kochen brauchst). Die Stufe 5 besagt, dass ein Nebensatz nicht auf den Hauptsatz folgt, sondern dass er den Hauptsatz unterbricht (z.B. wenn du kommst im Beispiel sodass, wenn du kommst, etwas zum Essen hast). Die höchste Stufe, Stufe 6, liegt vor, wenn die Äußerung ein erweitertes Partizipialattribut enthält. Jede Stufe ist komplexer als die vorausgehende, wobei das Wort komplexer psychisch zu deuten ist. Dementsprechend verlangt die Stufe 5 dem Lernenden eine anspruchsvollere mentale Verarbeitung ab, als dies mit der Stufe 4 der Fall ist.

Nachdem jeder minimalen satzwertigen Einheit die entsprechende Stufe zugeordnet worden ist, kann errechnet und unter Berücksichtigung der

17 Dies steht in Klammern, weil es sich bei diesem Teil um eine eigenständige minimale satzwertige Einheit handelt.

18 Vgl. Grießhaber: Zum Verfahren der Sprachprofilanalyse. 
Angaben in der Tabelle festgestellt werden, welche Stufe den Kenntnisstand des jeweiligen Probanden am besten kennzeichnet.

Die Analyse des oben angeführten Textteils sieht wie folgt aus:

\begin{tabular}{|l|c|c|c|c|c|c|c|}
\hline \multirow{2}{*}{ Minimale satzwertige Einheiten } & \multicolumn{7}{|c|}{ Stufen } \\
\cline { 2 - 8 } & \multicolumn{3}{|c|}{$\begin{array}{c}\text { komplexe } \\
\text { Strukturen }\end{array}$} & \multicolumn{2}{c|}{$\begin{array}{c}\text { einfache } \\
\text { Strukturen }\end{array}$} \\
\hline & $\mathbf{6}$ & $\mathbf{5}$ & $\mathbf{4}$ & $\mathbf{3}$ & $\mathbf{2}$ & $\mathbf{1}$ & $\mathbf{0}$ \\
\hline 1. Inzwischen werde ich einkaufen gehen & & & & $\times$ & & & \\
\hline $\begin{array}{l}\text { 2. und dir Schinken, Salaten, Milch und Eier im } \\
\text { Kühlschrank lassen, }\end{array}$ & & & & & & & \\
\hline 3. sodass, (wenn du kommst), etwas zum Essen hast. & & & $\times$ & & & & \\
\hline 4. wenn du kommst & & $\times$ & & & & & \\
\hline 5. In der Kuche befinden sich alle Haushaltsgeräte, & & & & $\times$ & & & \\
\hline 6. die du fürs Kochen brauchst. & & & $\times$ & & & & \\
\hline 7. Bügeleisen werde ich in Wohnzimmer lassen & & & & $\times$ & & & \\
\hline $\begin{array}{l}\text { 8. und eine Waschmaschine wirst du in dem Bad } \\
\text { finden. }\end{array}$ & & & & $\times$ & & & \\
\hline
\end{tabular}

Tabelle 1. Durchführung der Profilanalyse - ein Beispiel

Wie der Tabelle 1 entnommen werden kann, wird in der Profilanalyse auch das Verhältnis zwischen komplexen und einfachen Strukturen betrachtet, wobei die Stufen 0 bis 2 als einfach, alle anderen als komplex gewertet werden.

Wenn der ganze Text, aus dem die acht minimalen satzwertigen Einheiten stammen, analysiert wird, wird die Vorkommenshäufigkeit einzelner Strukturen errechnet. Im Text kommen insgesamt 36 minimale satzwertige Einheiten vor. Mit wie vielen Beispielen die einzelnen Stufen vertreten sind, zeigt die Tabelle 2.

\begin{tabular}{|c|c|c|c|c|c|c|c|}
\hline \multirow{3}{*}{ Minimale satzwertige Einheiten } & \multicolumn{7}{|c|}{ Stufen } \\
\hline & \multicolumn{4}{|c|}{$\begin{array}{l}\text { komplexe } \\
\text { Strukturen }\end{array}$} & \multicolumn{3}{|c|}{$\begin{array}{l}\text { einfache } \\
\text { Strukturen }\end{array}$} \\
\hline & 6 & 5 & 4 & 3 & 2 & 1 & $\mathbf{0}$ \\
\hline \multirow{3}{*}{ Ergebnis } & 0 & 1 & 11 & 11 & 4 & 6 & 3 \\
\hline & \multicolumn{4}{|c|}{23} & \multicolumn{3}{|c|}{13} \\
\hline & \multicolumn{4}{|c|}{$64 \%$} & \multicolumn{3}{|c|}{$36 \%$} \\
\hline
\end{tabular}

Tabelle 2. Ergebnisse der Profilanalyse - ein Beispiel

Die höchste Stufe, die mindestens dreimal in einem Text vorkommt, wird als die Stufe betrachtet, die der betreffende Proband zum Zeitpunkt der Datenerhebung erreicht hat, d.h. es wird das Prinzip des Mindestvor- 
kommens angewandt. ${ }^{19}$ Dabei ist es nicht wichtig, wie viele Belege es für die niedrigeren Stufen gibt. Die Untersuchungen haben gezeigt, dass die Beherrschung einer Struktur die Beherrschung aller niedrigeren Stufen (bei dem Probanden) impliziert, da die besprochenen Stufen sequentiell erworben werden. Die Profilanalyse dieses Textes zeigt einen überwiegenden Anteil an komplexen Strukturen. Der Proband, auf dessen schriftliche Produktion sich die Angaben in der Tabelle 2 beziehen, hat somit die Stufe 4 erreicht. Er ist ein fortgeschrittener Lernender, trotzdem kann sein Syntaxerwerb nicht als abgeschlossen betrachtet werden, denn Stufe 5 ist bei ihm nur einmal, Stufe 6 dagegen überhaupt nicht vorgekommen.

Neben dem Vorteil, dass die Analyse schnell durchgeführt werden kann, nachdem man die Methode erfasst und vollständig erlernt hat, ist sie auch insofern interessant, als sie zusätzliche Informationen bezüglich des Kenntnisstandes des Probanden liefert. Die Stufenermittlung gewährt Einsichten in andere Bereiche, z.B. in den der Wortschatzbeherrschung. Die erreichte Stufe der Grammatikkompetenz eines Probanden korreliert demnach mit seinen Kenntnissen des Wortschatzes: bessere Ergebnisse nach der Profilanalyse bedeuten bessere Wortschatzkenntnisse. ${ }^{20}$

\subsection{Ergebnisse}

Der Umfang der schriftlichen Produktionen unserer Probanden beläuft sich auf zwischen 112 und 321 Wörtern, durchschnittlich enthält der Text 173,6 Wörter. Die Anzahl der Sätze pro Text beläuft sich auf 8 bis 26, durchschnittlich enthält der Text 15,2 Sätze.

Für die schriftliche Produktion konnten die Probanden nach dem ECL-Raster maximal 25 Punkte gewinnen. Auf der Niveaustufe B1 befinden sich aber nur diejenigen, die zwischen 15 und 25 Punkten erzielten. 46 Probanden (81\%) sind demnach auf der Niveaustufe B1, 11 Probanden (19\%) haben diese Stufe noch nicht erreicht. Nur ein Proband erreichte alle 25 Punkte - seine schriftsprachliche Kompetenz mag über dem Niveau B1 liegen.

Im Bereich formelle Korrektheit erreichten die Probanden durchschnittlich 3,1 Punkte. Wie die Punktevergabe für diesen Aspekt ausgefallen ist, lässt sich der Tabelle 3 entnehmen.

19 Grießhaber: Beurteilung von Texten mehrsprachiger Schülerinnen und Schüler, S. 5.

20 Vgl. Grießhaber: Zum Verfahren der Sprachprofilanalyse; ders.: Sprachstandsdiagnose im kindlichen Zweitspracherwerb. 


\begin{tabular}{|c|c|c|}
\hline Punkte & Anzahl der Probanden & Prozentwerte \\
\hline 1 & 1 & 1,8 \\
\hline 2 & 9 & 15,8 \\
\hline 3 & 34 & 59,6 \\
\hline 4 & 11 & 19,3 \\
\hline 5 & 2 & 3,5 \\
\hline Insgesamt & 57 & 100,0 \\
\hline
\end{tabular}

Tabelle 3. Punkte für formelle Korrektheit im ECL-Test

Nach der Profilanalyse der schriftlichen Produktionen erreichte eine Mehrheit der Probanden (82,5\%) die Stufe 4, die Stufe 3 ist seltener vertreten, während die Stufen 2 und 5 eine Ausnahme darstellen. Genauere Angaben enthält die Tabelle 4.

\begin{tabular}{|c|c|c|}
\hline Erwerbsstufe & Anzahl der Probanden & Prozentwerte \\
\hline 2 & 1 & 1,8 \\
\hline 3 & 8 & 14,0 \\
\hline 4 & 47 & 82,5 \\
\hline 5 & 1 & 1,8 \\
\hline Insgesamt & 57 & 100,0 \\
\hline
\end{tabular}

Tabelle 4. Erwerbsstufe nach der Profilanalyse

Wie oben erwähnt, werden alle Probandentexte zum Zwecke der Profilanalyse in minimale satzwertige Einheiten zerlegt. Die Anzahl dieser Einheiten pro Text beläuft sich auf zwischen 18 und 52, wobei der Text im Durchschnitt 28,7 minimale satzwertige Einheiten beinhaltet. Wichtig für unsere Untersuchung ist das Verhältnis zwischen einfachen und komplexen syntaktischen Strukturen. Sogar 46\% aller minimalen satzwertigen Einheiten entfallen auf komplexe syntaktische Strukturen.

\begin{tabular}{|l|c|c|}
\hline & Anzahl & Prozentwerte \\
\hline Einfache syntaktische Strukturen (Stufen 0-2) & 873 & $54 \%$ \\
\hline Komplexe syntaktische Strukturen (Stufen 3-6) & 760 & $46 \%$ \\
\hline Anzahl der minimalen satzwertigen Einheiten & 1633 & $100 \%$ \\
\hline
\end{tabular}

Tabelle 5. Einfache und komplexe syntaktische Strukturen

Zur besseren Einsicht in die Syntaxkompetenz der Probanden wurden auch Korrelationen zwischen einigen Werten errechnet. 


\begin{tabular}{|c|c|c|c|c|c|}
\hline & & $\begin{array}{c}\text { Formelle } \\
\text { Korrektheit }\end{array}$ & $\begin{array}{l}\text { Erreichte } \\
\text { Stufe }\end{array}$ & $\begin{array}{l}\text { Komplexe } \\
\text { Strukturen }\end{array}$ & $\begin{array}{l}\text { Text- } \\
\text { umfang }\end{array}$ \\
\hline \multirow{3}{*}{$\begin{array}{l}\text { Formelle } \\
\text { Korrektheit }\end{array}$} & $\begin{array}{l}\text { Korrelation } \\
\text { nach Spearman }\end{array}$ & 1,000 & , 190 &, $305^{*}$ &, 233 \\
\hline & Sig. (2-seitig) & . & , 156 &, 021 & ,081 \\
\hline & $\mathrm{N}$ & 57 & 57 & 57 & 57 \\
\hline \multirow{3}{*}{$\begin{array}{l}\text { Erreichte } \\
\text { Stufe }\end{array}$} & $\begin{array}{l}\text { Korrelation } \\
\text { nach Spearman }\end{array}$ & , 190 & 1,000 &, $436^{* *}$ &, $345^{* *}$ \\
\hline & Sig. (2-seitig) & ,156 & . & ,001 &, 009 \\
\hline & $\mathrm{N}$ & 57 & 57 & 57 & 57 \\
\hline \multirow{3}{*}{$\begin{array}{l}\text { Komplexe } \\
\text { Strukturen }\end{array}$} & $\begin{array}{l}\text { Korrelation } \\
\text { nach Spearman }\end{array}$ &, $305^{*}$ &, $436^{* *}$ & 1,000 & ,226 \\
\hline & Sig. (2-seitig) &, 021 &, 001 & . & ,091 \\
\hline & $\mathrm{N}$ & 57 & 57 & 57 & 57 \\
\hline \multirow{3}{*}{ Textumfang } & $\begin{array}{l}\text { Korrelation } \\
\text { nach Spearman }\end{array}$ & 233 &, $345^{* *}$ & ,226 & 1,000 \\
\hline & Sig. (2-seitig) &, 081 & ,009 & ,091 & . \\
\hline & $\mathrm{N}$ & 57 & 57 & 57 & 57 \\
\hline
\end{tabular}

Tabelle 6. Korrelationen zwischen einzelnen Werten

Zwischen den Punkten für formelle Korrektheit und der nach der Profilanalyse errechneten Stufe ist keine Korrelation zu erkennen.

Es konnte eine schwache, statistisch signifikante Korrelation zwischen den Punkten für formelle Korrektheit und den Anteil an komplexen syntaktischen Strukturen nachgewiesen werden.

Eine mäßige, statistisch signifikante Korrelation ist zwischen der erreichten Stufe und dem Anteil an komplexen syntaktischen Strukturen zu beobachten, und eine schwache, aber statistisch signifikante Korrelation zwischen der erreichten Stufe und dem Textumfang.

\subsection{Interpretation der Ergebnisse}

Gemäß dem ECL-Test errreichten die meisten Probanden die Niveaustufe B1, nach der Profilanalyse die Stufe 4.

Die anhand der Analyse der schriftlichen Produktionen gewonnenen Ergebnisse deuten darauf hin, dass die meisten kroatischen Lernenden 
vor dem Abschluss der Mittelschule das Niveau B1 erreichen, während die fremdsprachlichen Kenntnisse einiger Lernender unter der Niveaustufe B1 liegen. Diese wenigen Lernenden weisen somit auch nach recht vielen Deutschstunden und nach der bestandenen Abiturprüfung in deutscher Sprache eine immer noch nur elementare Sprachbeherrschung auf. Wenn man bedenkt, dass erst die Niveaustufe B2 syntaktische Strukturen wie mehrfache Subordination und Partizipialkonstruktionen mit dem Partizip I und II umfasst, ${ }^{21}$ muss die Angabe, die höhere Stufe des kroatischen Abiturs entspreche der Niveaustufe B2, hinterfragt werden. ${ }^{22}$ Auf jeden Fall gilt sie nicht für die produktive Fertigkeit des Schreibens unserer Probandengruppe.

Nach der Profilanalyse haben die meisten Probanden die Stufe 4 erreicht. Nur ein Proband konnte der Stufe 5 zugeordnet werden, während 9 Probanden die Stufe 4 nicht erreichen konnten. Dies deutet darauf hin, dass die letztgenannten Probanden die deutsche Nebensatzstruktur nicht beherrschen. Obwohl die meisten Probanden die Stufe 4 erlangten, darf daraus nicht geschlossen werden, dass sie Nebensätze schlechthin beherrschen. Die Profilanalyse beschränkt sich auf topologische Besonderheiten, sodass sie im Zusammenhang mit der besprochenen Stufe die Angabe außer Betracht lässt, über welches Inventar an Nebensatzarten und Subjunktionen die Probanden in der Tat verfügen. Bspw. konnten in 370 Nebensätzen 14 verschiedene Subjunktionen verzeichnet werden, von denen dass und wenn am häufigsten vorkommen.

Einen besseren Einblick in den erreichten Stand der Probanden möchten wir uns verschaffen, indem wir einige einschlägige Beispiele aus ihren Produktionen anführen und kommentieren.

Die eingebetteten Nebensätze, wie der mit dem Relativpronomen die eingeleitete Nebensatz aus unserem Beispiel, kommen selten vor. Nur bei 18 Probanden konnte diese Stufe beobachtet werden:

(1) Und die aleinziehende Mutter die neben an wohnt hat ein kleines kind (Proband 39)

(2) aber du kannst bei meine Mutter die gleich um die Ecke wohnt deine Sachen waschen. (Proband 56)

Das folgende Beispiel ist vielleicht ein Beleg dafür, dass Syntaxwissen sequentiell erworben wird. Während die Teile des Verbs korrekt vonein- 
ander abgetrennt werden, befindet sich das Präfix noch immer nicht an letzter Stelle:

(3) Das hängt ab von dir. (Proband 23)

Genauso zeigt der Beleg (4), dass der Proband die zweite Stufe erreicht hat, aber noch nicht die dritte, denn der Rest des Prädikats (zur verfugung) besetzt nicht die letzte Position im Satz, wie dies standardsprachlich der Fall ist.

(4) Ich gebe dir meine Wohnung zur verfugung in der Zeit. (Proband 52)

Bei vielen Probanden konnten korrekte Inversionen beobachtet werden. Wenn man bedenkt, dass die Muttersprache unserer Probanden in diesem Punkt von der deutschen Sprache abweicht, zeugen solche Belege von einem erfolgreichen Syntaxerwerb:

(5) deshalb gibt es viel Gemüse und Milchprodukte (Proband 53)

Offen bleibt die Frage, wie ein Nebensatz, der eine korrekte Topologie aufweist, aber mit einem falschen Subjunktor eingeleitet wird, bewertet werden soll:

(6) Ob du ins Stadtzentrum gehen möchtest, gehe bitte nicht mit Verkehrsmitteln. (Proband 8)

Bei einem Probanden bspw. konnten insgesamt sechs Nebensätze verzeichnet werden. Drei davon sind topologisch richtig, aber sie werden mit dem falschen Subjunktor eingeleitet.

(7) Die Nachbarn können dir auch helfen, ob du sie brauchst. (Proband 18)

Aus diesen drei fehlerhaften Beispielen könnte man schließen, dass der Proband entweder die Bedeutung und Funktion von ob nicht gelernt hat oder dass er den Kontext nicht erkennen kann, in dem der Subjunktor wenn erforderlich ist. Die zweite Begründung ist wahrscheinlich falsch, denn die übrigen drei Nebensätze, die korrekt sind, enthalten die Subjunktoren wie, wenn und dass. Dies deutet darauf hin, dass der Proband den Kontext für wenn doch erkennen kann. Bisweilen kommen auch im Zusammenhang mit anderen syntaktischen Phänomenen Konstruktionen vor, die den Blick auf den Syntaxerwerb des Probanden eher verschleiern. So konnten bei ein und demselben Probanden folgende zwei Sätze gelesen werden:

(8) Was die Haushaltsgeräte betrifft, unten im Keller findest du die Waschmaschine und den Trockner. (Proband 11) 
(9) Was den Haushalt betrifft, möchte ich dich um einen Gefallen bitten. (Proband 11)

Im Beispiel (8) erscheint das Hauptsatzverb an falscher Stelle (...betrifft, unten im Keller findest $d u \rightarrow \ldots$ betrifft, findest $d u$ ). Da viele Sprecher solche was-Sätze nicht als Bestandteil des folgenden Teilsatzes ansehen, fühlt man sich geneigt zu sagen, dass der ganze Satz in topologischer Hinsicht korrekt ist. Da aber im gleichen Text dieselbe Nebensatzart noch einmal erscheint, dieses Mal aber mit dem Hauptsatzverb (möchte) an richtiger Stelle, ist es nicht einfach, den erreichten Stand des Probanden anhand der vorhandenen schriftlichen Produktion zu bewerten.

Die Profilanalyse unterscheidet die verschiedenen Abhängigkeitsgrade der Nebensätze nicht. So werden alle drei Nebensätze im folgenden Beispiel der Stufe 4 zugeordnet:

(10) Ich denke, das es dir gut bei mir wird und das du alles hast, was du brauchst. (Proband 35)

Die Profilanalyse ist nicht in der Lage, den Umstand wahrzunehmen, dass der was-Satz auf der zweiten Abhängigkeitsstufe liegt und dass der ganze Satz ein Beispiel für mehrfache Subordination ist. Aber u.E. ist es nicht übertrieben, bei Probanden, die solche Sätze produzieren, eine höhere Stufe des Syntaxerwerbs anzusetzen als bei Probanden, die ausschließlich Nebensätze ersten Grades produzieren.

Die Analyse hat gezeigt, dass die eingesetzte Methode tatsächlich die syntaktische Ebene misst und wenig über die kommunikative Kompetenz auszusagen vermag bzw. dass ein höherer Grad an syntaktischer Komplexität nicht unbedingt mit einem höheren Grad an kommunikativer Kompetenz korreliert:

(11) Ich hoffe, das du Obst und Salami magst. Fals nicht, hast du gegen über einen kleinen Supermarket. (Proband 38)

Die minimale Einheit »Fals nicht « wird als Stufe 0 aufgefasst. An diesem Beispiel lässt sich nicht ablesen, welche Grammatikkompetenz der betreffende Proband besitzt. Aber der Beleg widerspiegelt eine recht gute kommunikative Kompetenz.

Bei der Berücksichtigung aller schriftlichen Produktionen zeigt sich, dass 54\% aller minimalen satzwertigen Einheiten zu einfachen syntaktischen Strukturen gehören. Zu diesen gehören nur einfache Sätze mit dem Subjekt in Erstposition. Der Anteil komplexer Strukturen bei der schwächsten schriftlichen Produktion beläuft sich auf $17 \%$ und bei der besten auf $72 \%$, während dieser Wert durchschnittlich $46 \%$ beträgt. Diese Angabe verweist 
möglicherweise auf einen langsamen Syntaxerwerb. Die Interpretation hängt natürlich mit der Frage zusammen, ob bzw. inwiefern die Aufgabe und die Aufgabenstellung den Gebrauch von komplexen Strukturen bzw. Kreativität fördern. Diesbezüglich dürfen wir auch einen anderen Umstand nicht aus dem Auge verlieren: die Textlänge war vorgegeben. Wenn die Probanden längere Produktionen hätten schreiben dürfen, wären sie in der Lage gewesen, bestimmte Fragen tiefer zu elaborieren, was unserer Meinung nach den Gebrauch von komplexeren syntaktischen Strukturen gefördert hätte. Hinzu kommt, dass die Probandentexte im Kontext einer Prüfung entstanden sind. Dies ist ein psychologisches Moment, das eine entscheidende Rolle spielt. Dass wir bei den meisten Probanden die Stufe 4 identifiziert haben, mag also mit der Aufgabenstellung sowie mit den Umständen zusammenhängen, unter denen die Texte verfasst wurden.

Beim statistischen Vergleich der Ergebnisse, die die ECL-Bewertung und die Anwendung der Profilanalyse hervorgebracht haben, zeigt sich, dass die Punkte für formelle Korrektheit nicht mit der errechneten Stufe, aber dafür mit dem Prozentwert für komplexe Strukturen korrelieren. Mit anderen Worten konnte bei den Probanden, die mehrere Punkte für formelle Korrektheit gewannen, ein höherer Anteil an komplexen Strukturen beobachtet werden. Dass die Punkte für formelle Korrektheit nicht mit der errechneten Stufe korrelieren, könnte vielleicht mit dem Konstrukt erklärt werden, das gemessen wird. Wie gesagt, die Profilanalyse beschränkt sich nur auf die syntaktische Ebene, während formelle Korrektheit auch die morphologische Ebene einbezieht. Werden die Ergebnisse zweier Analysen den Angaben zum Textumfang gegenübergestellt, kann eine höhere syntaktische Komplexität bei längeren schriftlichen Produktionen festgestellt werden. Umfangreichere Texte enthalten somit keinesfalls eine größere Anzahl an einfachen Sätzen, sondern sie weisen eine höhere syntaktische Komplexität auf.

Das Verhältnis zwischen den beobachteten Merkmalen bzw. Werten lässt sich folgendermaßen darstellen:

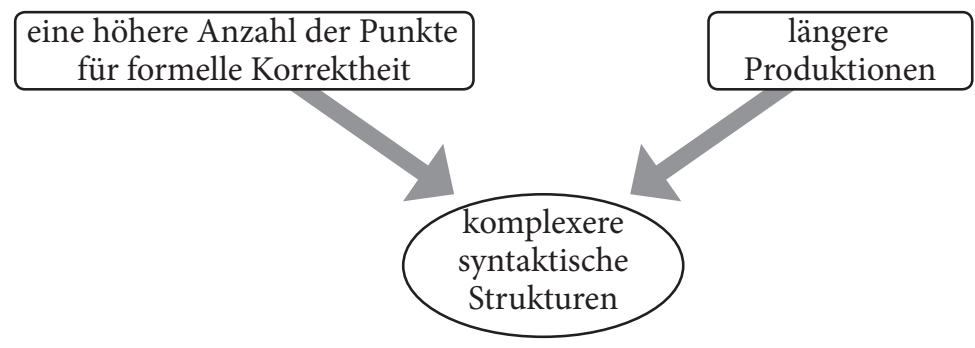


Die Pfeile sollen dabei signalisieren, dass eine bessere Bewertung der formellen Korrektheit und ein größerer Textumfang auf eine höhere syntaktische Komplexität der schriftlichen Produktion hindeuten.

Dem Anschein nach fallen die erreichte Stufe (Stufe 4) und die Beschreibung der Grammatikkompetenz, wie sie in den betreffenden kroatischen Lehrplänen vorhanden ist, auseinander. Zwar zeigen die empirischen Ergebnisse, dass die meisten Probanden in ihren schriftlichen Produktionen Nebensätze souverän gebrauchen, obwohl das Inventar an Nebensatzarten und Subjunktionen, über welches sie verfügen, nicht gerade hoch ist, aber unterbrochene Sätze mit eingebetteten Nebensätzen sowie erweiterte Partizipialattribute beherrschen sie nicht. Auch die Verwendung des Konjunktivs und der irrealen Nebensätze scheint relativ bescheiden zu sein. Offiziell müssten unsere Probanden die genannten Strukturen richtig verstehen und gebrauchen können. Einige dieser Strukturen werden explizit genannt, ${ }^{23}$ während die anderen aus den Sprachfunktionen ${ }^{24}$ abgeleitet werden können, die kroatischen DaF-Lernenden bekannt sein sollten. Die Umstände, unter denen die Probanden ihre Texte verfasst haben, wirkten sich möglicherweise auf den Gebrauch von komplex(er)en Strukturen negativ aus, worauf im obigen Text schon verwiesen wurde.

\section{Abschließende Bemerkungen}

Die vorliegende Untersuchung hat aufschlussreiche Befunde bezüglich der Grammatikkompetenz kroatischer DaF-Lernender sowie der Angemessenheit der Profilanalyse als Methode hervorgebracht. Grob gesagt, wurde hierbei empirisch nachgewiesen, dass die Entwicklung der Grammatikkompetenz etwas langsamer verläuft, als dies unsere Lehrpläne vorsehen. Allem Anschein nach widerspiegelt sich hier die gedächtnispsychologische Bedingtheit des Fremdsprachenlernens, die in den Arbeiten zur Profilanalyse angesprochen wird. Die Untersuchung hat aber auch ganz eindeutig gezeigt, welche syntaktischen Strukturen die Probanden beherrschen. Hoffentlich werden diese Ergebnisse von all jenen wahrgenommen, in deren Zuständigkeitsbereich die Gestaltung der Lehrpläne für Deutsch als Fremdsprache fällt.

In der Untersuchung konnten wir beobachten, dass die Profilanalyse zwar leicht anwendbar ist, dass sie aber mit einer weiteren Methode oder 
einem Raster ergänzt werden sollte, falls etwas differenziertere Angaben zur Grammatikkompetenz gewonnen werden sollen. Vor allem springt ins Auge, dass sie die syntaktische Komplexität zum Teil gut wahrnimmt (z.B. erweiterte Partizipialattribute), zum Teil aber überhaupt nicht zu registrieren vermag (z.B. mehrfache Subordination).

\section{Literaturverzeichnis}

Bachman, Lyle F.; Palmer, Adrian S.: The Construct Validation of Some Components of Communicative Proficiency. »TESOL Quarterly« 16.4 (1982), S. 449-467.

Bachman, Lyle F.; Palmer, Adrian S.: Language Assessment in Practice. Oxford u.a.: Oxford University Press 2010.

Bachman, Lyle F.; Palmer, Adrian S.: Language Testing in Practice: Designing and Developing Useful Language Tests. Oxford u.a.: Oxford University Press 1996.

Bagarić Medve, Vesna: Komunikacijska kompetencija. Uvod u teorijske, empirijske i primijenjene aspekte komunikacijske kompetencije u stranom jeziku. Osijek: Filozofski fakultet Sveučilišta Josipa Jurja Strossmayera 2012.

Canale, Michael; Swain, Merrill: Theoretical Bases of Communicative Approaches to Second Language Teaching and Testing. "Applied Linguistics" 1.1 (1980), S. 1-47.

Canale, Michael; Swain, Merrill: A Theoretical Framework for Communicative Competence. In: The Construct Validation of Tests of Communicative Competence. Hgg. Adrian S. Palmer, Peter J. M. Groot, George A. Trosper. Washington: TESOL 1981, S. 31-36.

Celce-Murcia, Marianne; Dörnyei, Zoltán; Thurrell, Sarah: Communicative Competence: A Pedagogically Motivated Model with Content Specifications. "Issues in Applied Linguistics« 6.2 (1995), S. 5-35.

Cohen, Andrew D.: Assessing Language Ability in the Classroom. Boston, Massachusetts: Heinle and Heinle Publishers 1994.

Diehl, Erika: Schulischer Grammatikerwerb unter der Lupe: Das Genfer DiGS-Projekt. »Bulletin suisse de linguistique appliquée« 70 (1999), S. 7-26.

Diehl, Erika et al.: Grammatikunterricht: Alles für der Katz? Untersuchungen zum Zweitspracherwerb Deutsch. Niemeyer: Tübingen 2000.

ECL-Informationsblatt = ECL Konzorcium Nemzetközi Titkársága és az akkreditált ECL nyelvvizsgarendszer magyarországi központja. Tájékoztató vizsgázók és felkészitő tanárok számára az ECL nyelvvizsgáról. Pécs: Pécsi tudományegyetem 2013.

Ellis, Rod: Current Issues in the Teaching of Grammar. An SLA Perspective. »TESOL Quarterly« 40.1 (2006), S. 83-107.

Ellis, Rod: Principles of Instructed Second Language Acquisition. In: CALdigest (2008): S. 1-6. <http://moodle2.unifr.ch/pluginfile.php/166786/mod_resource/content/2/ Texte/03d_Ellis_2008_Instructed2ndLangFinalWeb.pdf> (Zugriff: 5.11.2014).

Grießhaber, Wilhelm: Beurteilung von Texten mehrsprachiger Schülerinnen und Schüler. »leseforum.ch - Online-Plattform für Literalität« 3 (2014), S. 1-19.

Grießhaber, Wilhelm: Sprachstandsdiagnose im kindlichen Zweitspracherwerb: Funktionalpragmatische Fundierung der Profilanalyse (2006) <http://spzwww.uni-muenster.de/ griesha/pub/tprofilanalyse-azm-05.pdf> (Zugriff: 20.4.2015). 
Grießhaber, Wilhelm: Zum Verfahren der Sprachprofilanalyse (Grießhaber 2002-2005). $<$ http://spzwww.uni-muenster.de/griesha/dpc/profile/profilhintergrund.html $>$ (Zugriff: 20.4.2015).

Ispitni katalog za državnu maturu u školskoj godini 2014./2015. Njemački jezik. Nacionalni centar za vanjsko vrednovanje obrazovanja. <http://dokumenti.ncvvo.hr/Ispitni_katalozi_14-15/Hrvatski/IK-njem.pdf> (Zugriff: 20.4.2015).

Pon, Leonard: Grammatisches Wissen, Fremdsprachenunterricht und Lehrwerke - Zur Qualität von Grammatikübungen in zwei kroatischen DaF-Lehrwerken. "Linguistik online« 69.7 (2014), S. 37-68.

Sanchez, Laura: Against >Canonical Word Order: Evidence of Basic Word Order Transfer at the Initial State of LnA. »Newcastle Working Papers in Linguistics« 17 (2011), S. 220-236.

Savignon, Sandra J.: Communicative Competence: Theory and Classroom Practice. Texts and Contexts in Second Language Learning. Reading, Massachusetts: Addison-Wesley Publishing Company 1983.

Trim, John; North, Brian; Coste, Daniel; Sheils, Joseph: Gemeinsamer europäischer Referenzrahmen für Sprachen: lernen, lehren, beurteilen. Berlin, Wien: Langenscheidt 2001. 